\title{
Effect of iron supplementation on mental and motor development in children: systematic review of randomised controlled trials
}

\author{
HPS Sachdev ${ }^{1, *,} \dagger$, Tarun Gera ${ }^{2}$ and Penelope Nestel ${ }^{3}$ \\ 'Division of Clinical Epidemiology, Department of Pediatrics, Maulana Azad Medical College, New Delhi 110002 , \\ India: ${ }^{2}$ SL Jain Hospital, New Delhi 110 052, India: ${ }^{3}$ HarvestPlus, International Food Policy Research Institute, \\ 2033 K Street NW, Washington, DC 20006-1002, USA
}

Submitted 30 June 2004: Accepted 22 September 2004

\begin{abstract}
Objective: To evaluate the effect of iron supplementation on mental and motor development in children through a systematic review of randomised controlled trials (RCTs).

Data sources: Electronic databases, personal files, hand search of reviews, bibliographies of books, abstracts and proceedings of international conferences.

Review methods: RCTs with interventions that included oral or parenteral iron supplementation, fortified formula milk or cereals were evaluated. The outcomes studied were mental and motor development scores and various individual development tests employed, including Bayley mental and psychomotor development indices and intelligence quotient.

Results: The pooled estimate (random effects model) of mental development score standardised mean difference (SMD) was 0.30 (95\% confidence interval (CI) 0.15 to $0.46, P<0.001 ; P<0.001$ for heterogeneity). Initial anaemia and iron-deficiency anaemia were significant explanatory variables for heterogeneity. The pooled estimate of Bayley Mental Development Index (weighted mean difference) in younger children $(<27$ months old) was 0.95 (95\% CI -0.56 to $2.46, P=0.22$; $P=0.016$ for heterogeneity). For intelligence quotient scores ( $\geq 8$ years age), the pooled SMD was 0.41 (95\% CI 0.20 to $0.62, P<0.001 ; P=0.07$ for heterogeneity). There was no effect of iron supplementation on motor development score (SMD 0.09, 95\% CI -0.08 to $0.26, P=0.28 ; P=0.028$ for heterogeneity).

Conclusions: Iron supplementation improves mental development score modestly. This effect is particularly apparent for intelligence tests above 7 years of age and in initially anaemic or iron-deficient anaemic subjects. There is no convincing evidence that iron treatment has an effect on mental development in children below 27 months of age or on motor development.
\end{abstract}

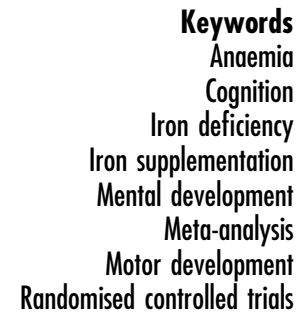

Animal studies have provided a number of possible mechanisms through which iron deficiency can leave an imprint on the developing brain ${ }^{1-4}$. Most observational studies in children have found associations between irondeficiency anaemia (IDA) and poor cognitive and motor development and behavioural problems ${ }^{5,6}$. Longitudinal studies consistently indicate that children who were anaemic in infancy continue to have poorer cognition, school achievement and more behaviour problems into middle childhood $^{6}$. However, the possible confounding effects of environmental factors, particularly poor socio-economic

†Correspondence address: E-6/12 Vasant Vihar, New Delhi 110 057, India. background, prevent causal inferences from being made. Furthermore, there is no convincing evidence that iron therapy can significantly improve psychomotor development and cognitive function in children under the age of 3 years with IDA $^{7}$. This may be related to confounding by environmental factors and a possible irreversible effect of iron deficiency on the developing brain, particularly on the dopamine receptors and the myelin tissue $\mathrm{e}^{3,4}$. It is important to evaluate the effect of iron administration on mental and motor development in children, including those in older age groups, to provide clarity about realistic expectations from iron supplementation and fortification efforts. We therefore conducted a systematic review to determine the effect of iron supplementation on mental and motor development in children. 


\section{Methods}

\section{Searching}

We searched computerised bibliographic medical databases, including Medline (1966 to March 2003), the Cochrane controlled trials register, Embase, IBIDS and Healthstar. We also reviewed reference lists of identified articles and hand searched reviews, bibliographies of books and abstracts and proceedings of international conferences or meetings. Donor agencies, 'experts' and authors of recent iron supplementation trials were contacted to identify any additional or ongoing trials. The title and abstract of the trials identified in the computerised search were scanned to exclude studies that were obviously irrelevant. We reviewed the full texts of the remaining studies and identified trials that fulfilled the inclusion criteria. To avoid publication bias, we included published and unpublished trials.

\section{Selection criteria}

To be included, trials had to:

- be randomised placebo-controlled trials - except for those in which iron was given parenterally, in which case trials did not have to be placebo-controlled because it would be difficult to administer a similar placebo;

- investigate iron supplementation through the oral or parenteral route or as formula milk or cereals fortified with iron; and

- evaluate one or more developmental indicators (psychomotor development, cognition, mental development, intelligence quotient (IQ), school performance) as an outcome measure.

We also included studies in which other micronutrients and drugs were simultaneously administered if the only difference between the study and the control groups was iron supplementation.

\section{Validity assessment}

We assessed the quality of trials using recommended criteria $^{8,9}$. Concealment of allocation was classed as adequate, unclear, inadequate, or not used. To assess attrition we classified studies by percentage of participants lost to follow-up ( $<3 \%, 3-9.9 \%, 10-19.9 \%$ and $\geq 20 \%)$. Blinding was classified as double blinding, single blinding, no blinding, or unclear.

\section{Data abstraction}

We used pre-formed questionnaires to abstract data. The data included in this review were derived from the published papers or were provided by the authors. If required, and wherever possible, we contacted the authors for clarifications. T.G. abstracted all data.

\section{Quantitative data synthesis}

In studies with two or more iron intervention groups (different dosage or administration regimes) and a single control group, the sample size of the control group was divided equally between the number of intervention groups while retaining the same value for the change in outcome and its standard deviation (SD). This was done to avoid multiple counting of the control group (Oxman AD, personal communication, 2003; Deeks J, personal communication, 2003). Thus, some trials contributed more than one analytic component for statistical computations.

In computing pooled estimates, we required sample size, mean change in development score from the beginning to the end of the intervention and the SD of this change in the intervention and control groups. The following principles were used for derivations if actual variables were not stated.

1. In a group, the lower of the two stated sample sizes at the beginning or end of a trial was assumed to be the sample size for the change.

2. Wherever feasible, SD was back-calculated from the stated standard errors, $t$ or $P$ values.

3. Wherever not stated, the mean change in development score was computed as the difference of mean postand pre-intervention scores.

4. Wherever not stated, the mean age of subjects was computed as the average of the stated range.

The SD for the change in development scores was available or could be back-calculated from only a few studies. For the rest, this SD was computed assuming correlations of 0.5 , and 0 (independent) between the preand post-test variances ${ }^{10}$. Considering the number of assumptions and computations involved, and to be confident about the interpretation, three types of pooled estimates were calculated for each development score. In two, the change SD for values that were missing or could not be back-calculated were computed with the assumption of a correlation $(P)$ of 0.5 or of independence. For the third, the post-intervention scores and their respective SDs were used.

The presence of publication bias in the extracted data was evaluated by funnel plots ${ }^{11}$. We used the METABIAS command in STATA software to perform statistical tests for funnel plot asymmetry ${ }^{12}$. The pooled estimates of the weighted mean difference (WMD) of the evaluated change in outcome score between the control and intervention group were calculated by both fixed effects and random effects model assumptions using the METAN command in STATA software ${ }^{12}$. Where different outcome scoring scales were used, the standardised-weighted mean difference (SMD) was used. We report primarily random effects estimates because most of the pooled results obtained were statistically heterogeneous.

We carried out pre-specified stratified analyses for quality of methods; age of subjects; route of iron 
administration (parenteral, oral supplement or food fortification); duration of supplementation; baseline haemoglobin $(\mathrm{Hb})$ concentration in the supplemented group; and iron status of the study population. The contribution of these variables to heterogeneity was also explored by meta-regression using the METAREG command in STATA software with the restricted maximum likelihood option ${ }^{12}$.

\section{Results}

\section{Trial flow}

We identified 32 randomised controlled trials that were potentially eligible ${ }^{13-40}$. Fifteen studies were ineligible (Fig. 1). We therefore evaluated 17 trials in this systematic review: 16 published and one unpublished (Kimmons G, Moffatt MEK, Longstaffe S, Whalen-Besant J. Short term effects of intra-muscular iron on the behaviour of irondeficient children: a clinical trial).

\section{Study characteristics}

Table 1 depicts the baseline characteristics of the included trials. The studies were almost equally distributed between developed and developing countries (seven in Asia, three in Europe, three in North America, two in South America, one in Africa, location of one not clear). Most of the studies $(11 / 17)$ were conducted in infants and toddlers, while in six trials older children were evaluated. In four studies the intervention lasted less than 2 weeks, while nine trials intervened for 4 months or longer. In most reports the subjects received iron supplements in the form of oral medicinal iron (12/17), two studies used fortified foods and three trials administered iron parenterally. In younger children, the developmental aspect studied were mainly the Bayley indices for mental and psychomotor development (9/17). Other studies used motor and language scores; discrimination learning, oddity learning and Peabody Picture Vocabulary Tests (PPVT); cognition score, visual recall, mazes, clerical task; Denver Development Screening Test (DDST); and IQ and school performance. The studies were grouped and analysed for two parameters, namely mental/intelligence scores and motor development indices. In addition, to retain a certain degree of homogeneity, individual analyses were also done for Bayley's indices, IQ scores, psychomotor scores and school performance.

\section{Quantitative data synthesis}

\section{Mental development}

Mental development score (MDS). This nomenclature refers to a logical combination of different tests that assess the same aspect of mental development, namely the Bayley Mental Development Index (MDI), Stanford Binet Test, PPVT, IQ and cognition scores. Fifteen studies (Table 1) were included in this analysis.

The funnel plot was symmetrical (Fig. 2) with no evidence of publication bias by Egger $(P=0.694)$ or

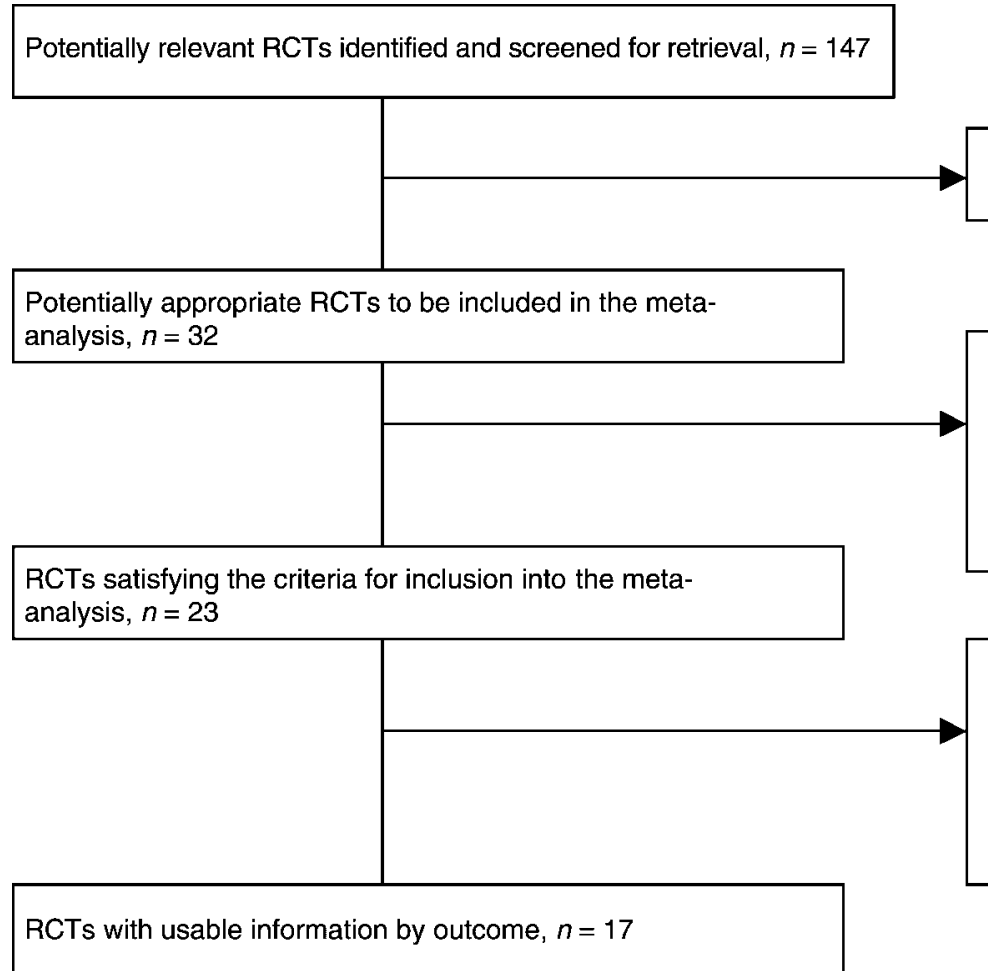

Obviously irrelevant studies excluded, $n=115$

Non RCTs: $2(18,29)$

Other drugs/micronutrients given with

iron: $4(21,23,25)$

Differernt milk or caloric consumption

by control and placebo: 3 (26-28)

Fig. 1 Flow chart depicting the trial flow for selection of randomised controlled trials (RCTs) to be included in the meta-analysis (reference numbers in parentheses) 


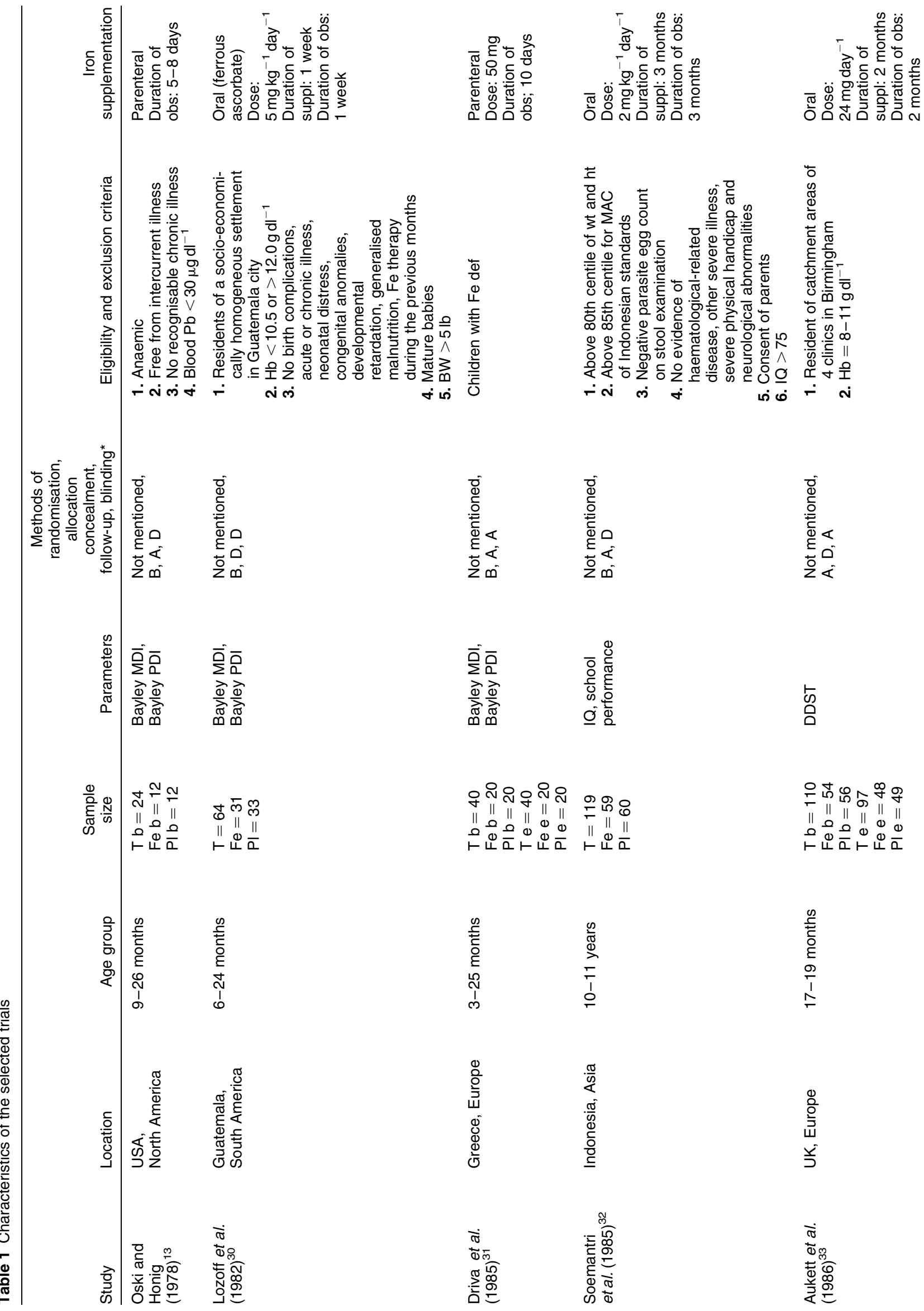


Iron supplementation and development in children

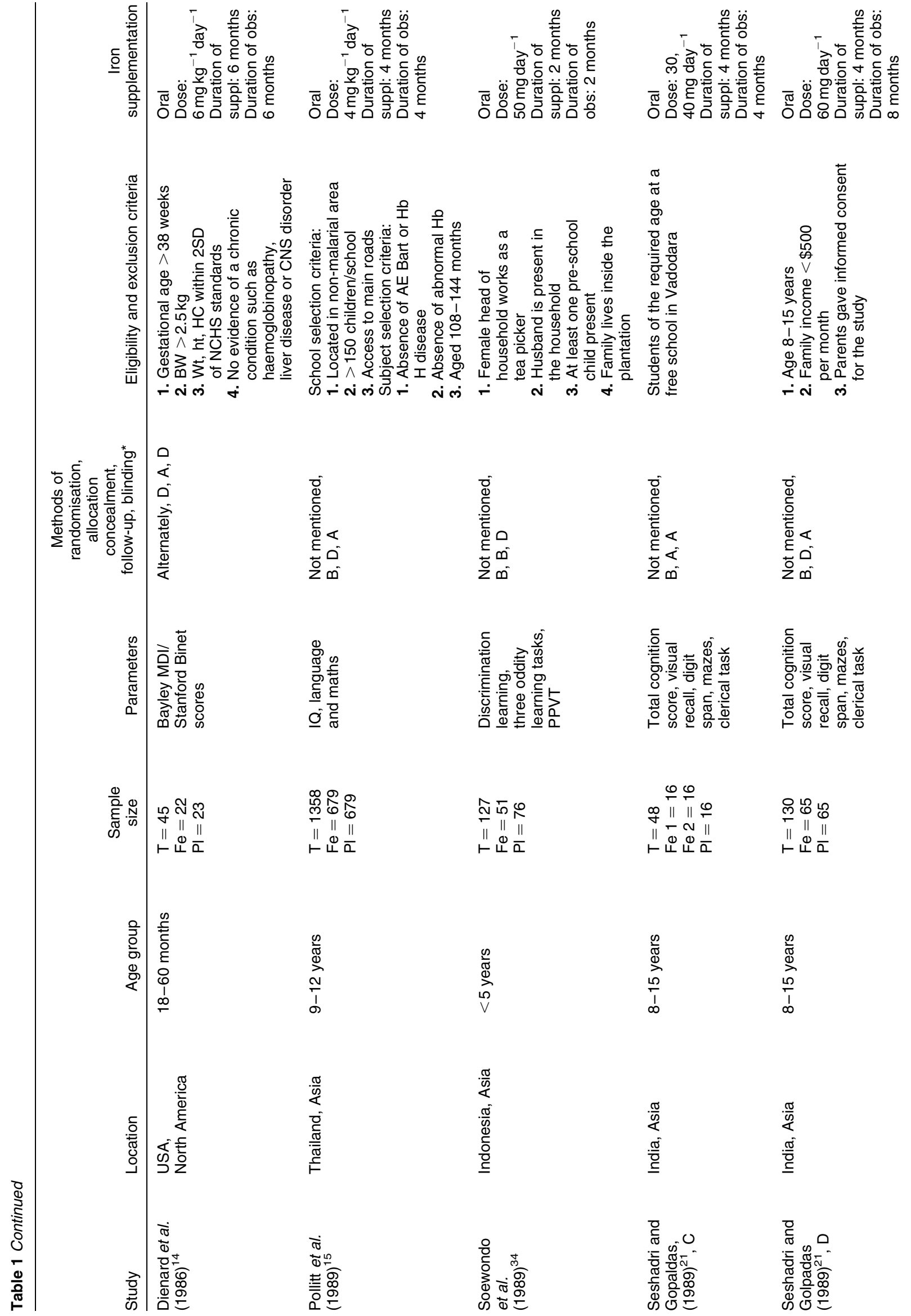




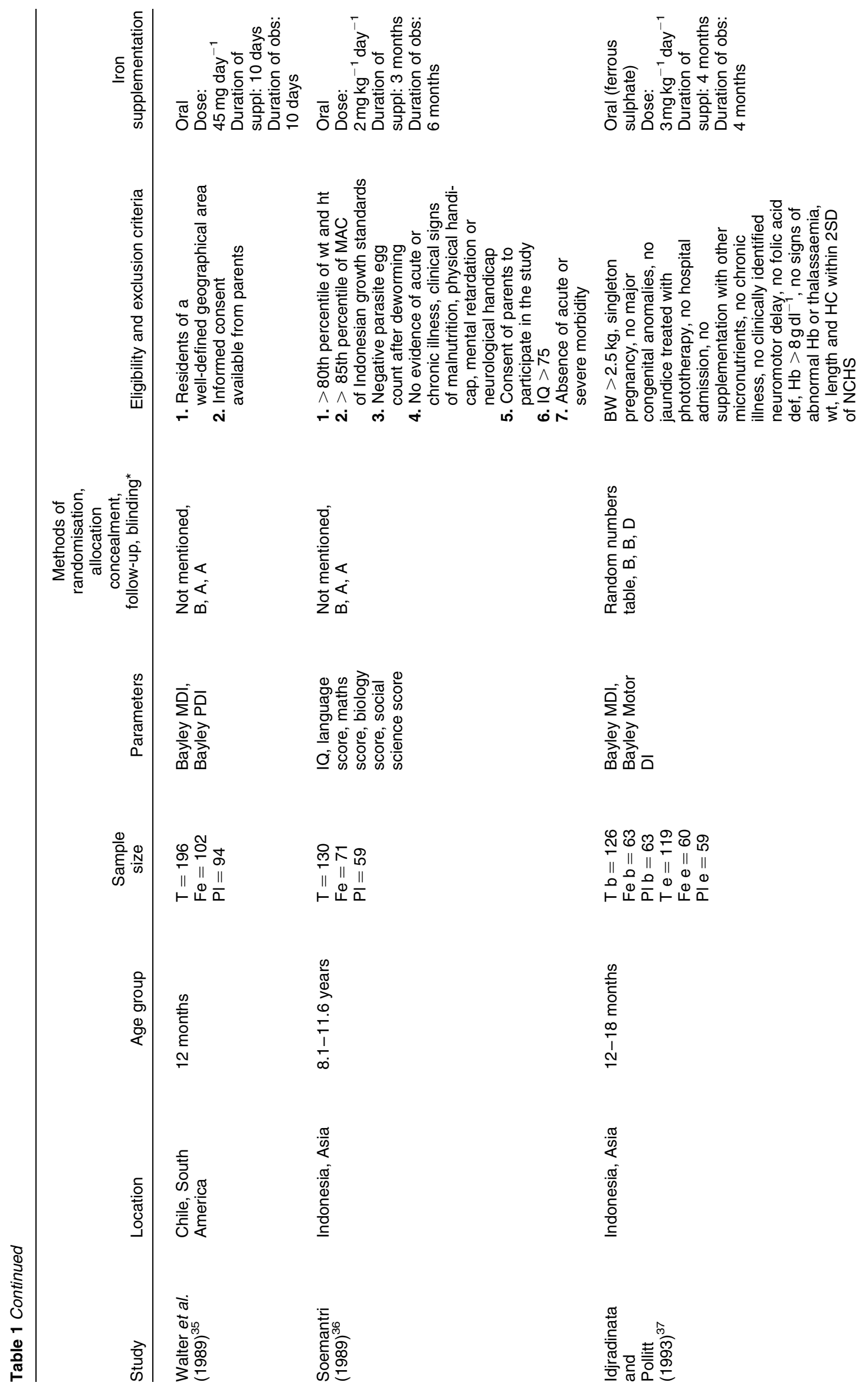


Iron supplementation and development in children

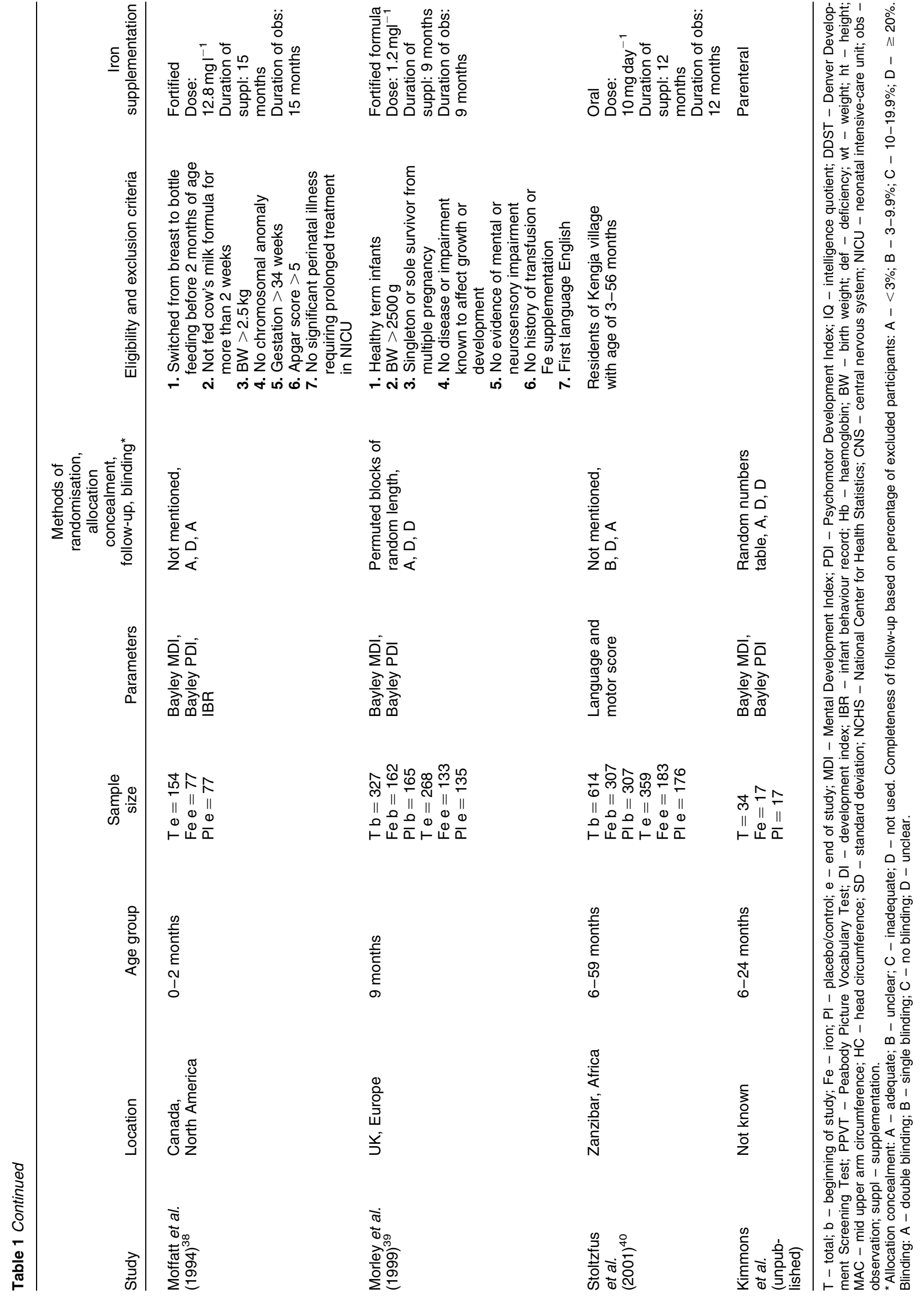




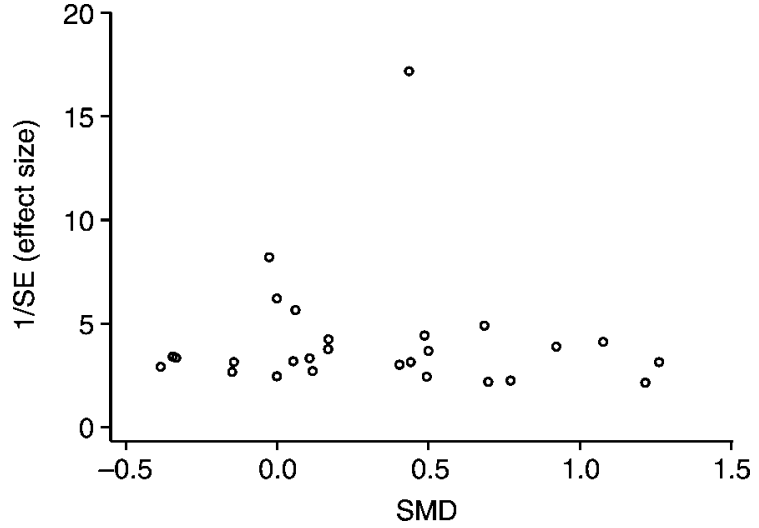

Fig. 2 Funnel plot of extracted studies for mental development score with unknown standard deviations derived under the assumption $P=0.5$. SE - standard error; SMD - standardised mean difference

Begg $(P=0.453)$ methods. We collected data on 2827 children, 1412 of whom received iron and 1415 placebo (Table 2). The pooled estimate (SMD) of the post-pre test difference in MDS following iron supplementation was 0.30 (95\% confidence interval (CI) 0.15 to 0.46 ; $P<0.001$ (Fig. 3, Table 3); test for heterogeneity $Q=72.05, P<0.001)$. The results were similar when SDs were calculated assuming $P=0.5$, assuming independence and with post-test scores. Sensitivity analysis suggested that greater benefits were associated with oral route of supplementation, longer duration of iron therapy ( $>1$ month), older age $(>5$ years) and lower baseline $\mathrm{Hb}$ and iron status. Meta-regression did not show any consistent association between the effect on MDS and duration of supplementation or age; however, lower baseline $\mathrm{Hb}$ and initially iron-deficient anaemic subjects were significant predictors of a positive effect of iron supplementation (Table 4).

Individual mental development tests (Table 5). Eight studies (references 13, 30, 31, 35, 37-39 and Kimmons et al., unpublished) on younger children $(<27$ months of age) assessed Bayley MDI. The pooled estimate (WMD) was 0.95 (95\% CI -0.56 to 2.46) and was not statistically significant $(P=0.217)$. On stratified analysis, iron-deficient anaemic children showed greater improvement in MDI scores vis-à-vis the control group $(\mathrm{WMD}=3.77,95 \% \mathrm{CI}-0.50$ to 8.04$)$, but the difference was not statistically significant $(P=0.08)$. On metaregression, when controlled for all other variables, IDA (vs. others) was a significant predictor of a beneficial response (WMD $=2.76,95 \%$ CI 0.26 to $5.25, P=0.03$ ). On combining a trial ${ }^{14}$ using both the Bayley MDI and the Stanford Binet Test with these eight studies, the pooled estimate was not statistically significant (SMD $=0.12,95 \%$ CI -0.07 to $0.30, P=0.219)$.

Four trials ${ }^{15,21,36}$ evaluated IQ scores in children aged 8 years or more. The pooled SMD was 0.41 ( $95 \%$ CI 0.20 to $0.62)$, which was significant $(P<0.001)$. Children with initial anaemia $\left(\mathrm{Hb}<11 \mathrm{~g} \mathrm{dl}^{-1}\right)$ and IDA had a greater improvement, but this was not confirmed on metaregression.

Two trials ${ }^{21}$ reported the effect of iron supplementation on the individual components of the total cognition score used. The response to iron supplementation on each of these components was pooled and all four parameters (digit span, visual recall, mazes and clerical tests) showed an effect by one or more of the computational methods employed; however, the effect was consistently significant for mazes only. The studies evaluating the effect of iron supplementation on linguistic (three trials) ${ }^{15,36,40}$ and mathematical (two trials) ${ }^{15,36}$ capabilities did not show any significant benefit of iron supplementation.

\section{Motor development}

Among the 10 trials evaluating motor development (references 13, 30, 31, 33, 35, 37-40 and Kimmons et al., unpublished), eight used the Bayley Psychomotor Development Index (PDI), one assessed psychomotor development through DDST $^{33}$ and one used a physical activity score $^{40}$. The funnel plot (Fig. 4) was symmetrical with no evidence of publication bias by Begg $(P=0.921)$ and Egger $(P=0.826)$ tests. We collected data on 1246 children; 630 received iron and 616 placebo. The pooled SMD with missing change SDs calculated with the assumption $P=0.5$ (Fig. 5) was 0.09 (95\% CI -0.08 to $0.26, P=0.28$; test for heterogeneity $Q=25.69, P=0.028$ ). Comparable pooled estimates were obtained with SDs computed under the assumption of independence $(0.09,95 \% \mathrm{CI}-0.08$ to 0.26 , $P=0.305$; test for heterogeneity $Q=25.49, P=0.03)$ and with post-test scores $(0.12,95 \% \mathrm{CI}-0.08$ to $0.32, P=0.24$; test for heterogeneity $Q=28.92, P=0.007)$. Sensitivity and meta-regression (Table 6) analyses indicated that quality of the study, route of supplementation, duration of supplementation, baseline $\mathrm{Hb}$ and iron status were not significant predictors of SMD. Similarly, there was no benefit of iron supplementation on psychomotor development and specifically Bayley PDI scores (Table 5).

\section{Discussion}

The results from our analysis of these studies show that iron supplementation improves the mental development score of children marginally (SMD $0.30 ; 95 \%$ CI 0.15 to 0.46 ) but significantly $(P<0.001)$. The benefits were greater among initially anaemic or iron-deficient anaemic subjects and these traits were significant explanatory variables for heterogeneity. In younger children $(<27$ months old $)$ invariably Bayley MDI was evaluated, which did not show any significant improvement with iron supplementation but there was a suggestion of benefit in those with initial IDA. In the four trials involving children over 7 years old, iron administration resulted in a significant improvement in 
Table 2 Data extracted from included studies with missing change standard deviation (SD) computed with the assumption $P=0.5$

\begin{tabular}{|c|c|c|c|c|c|c|c|}
\hline \multirow[b]{2}{*}{ Author (reference) } & \multirow[b]{2}{*}{ Outcome } & \multicolumn{3}{|c|}{$\begin{array}{l}\text { Change in iron } \\
\text { supplement group }\end{array}$} & \multicolumn{3}{|c|}{$\begin{array}{l}\text { Change in } \\
\text { placebo group }\end{array}$} \\
\hline & & Number & Mean & SD & Number & Mean & SD \\
\hline \multicolumn{8}{|l|}{ Mental development score } \\
\hline Kimmons (unpublished) & Bayley MDI & 17 & 4.10 & 3.90 & 17 & 5.60 & 3.90 \\
\hline Oski (13) & Bayley MDI & 12 & 13.58 & 15.15 & 12 & 6.08 & 15.15 \\
\hline Lozoff $1(30)$ & Bayley MDI & 12 & 5.50 & 7.40 & 12 & 5.50 & 7.40 \\
\hline Lozoff 2 (30) & Bayley MDI & 19 & 5.50 & 7.40 & 21 & 5.10 & 7.40 \\
\hline Driva (31) & Bayley MDI & 20 & 7.00 & 9.50 & 20 & 2.80 & 9.50 \\
\hline Soemantri B1 (32) & IQ & 43 & 3.64 & 4.00 & 35 & -0.67 & 4.00 \\
\hline Soemantri B2 (32) & IQ & 16 & -0.29 & 4.00 & 25 & 0.28 & 4.00 \\
\hline Dienard (14) & $\begin{array}{l}\text { Bayley MDI and } \\
\text { Stanford Binet Test }\end{array}$ & 22 & 1.50 & 16.50 & 23 & 7.00 & 16.50 \\
\hline Walter 1 (35) & Bayley MDI & 24 & 8.60 & 5.40 & 15 & 6.70 & 3.20 \\
\hline Walter 2 (35) & Bayley MDI & 66 & 8.90 & 3.40 & 61 & 8.70 & 3.20 \\
\hline Walter 3 (35) & Bayley MDI & 12 & 8.70 & 3.50 & 18 & 8.30 & 3.30 \\
\hline Seshadri $\mathrm{Ca}(21)$ & Total cognition score & 16 & 2.88 & 3.61 & 8 & 0.09 & 3.61 \\
\hline Seshadri Cb (21) & Total cognition score & 16 & 4.82 & 3.89 & 8 & 0.09 & 3.89 \\
\hline Seshadri D1 (21) & Total cognition score & 36 & 6.00 & 5.74 & 45 & 3.20 & 5.74 \\
\hline Seshadri D2 (21) & Total cognition score & 10 & 5.70 & 3.36 & 10 & 3.35 & 3.36 \\
\hline Soemantri 1 (36) & IQ & 37 & 1.50 & 6.38 & 35 & 0.41 & 6.38 \\
\hline Soemantri 2 (36) & IQ & 34 & 1.05 & 6.79 & 24 & -0.10 & 6.79 \\
\hline Pollitt 1 (15) & IQ & 51 & 4.00 & 5.84 & 50 & 0.00 & 5.84 \\
\hline Pollitt 2 (15) & IQ & 23 & 4.00 & 5.80 & 24 & 6.00 & 5.80 \\
\hline Pollitt 3 (15) & IQ & 605 & 5.00 & 2.29 & 605 & 4.00 & 2.29 \\
\hline Soewondo 1 (34) & PPVT & 27 & 6.70 & 8.60 & 43 & -1.23 & 8.60 \\
\hline Soewondo 2 (34) & PPVT & 24 & 6.05 & 8.60 & 33 & 1.75 & 8.60 \\
\hline Idjradinata 1 (37) & Bayley MDI & 24 & 19.30 & 14.90 & 23 & 0.50 & 14.90 \\
\hline Idjradinata 2 (37) & Bayley MDI & 14 & 5.30 & 14.90 & 14 & 7.50 & 14.90 \\
\hline Idjradinata 3 (37) & Bayley MDI & 22 & 3.70 & 14.90 & 22 & 2.10 & 14.90 \\
\hline Mofatt (38) & Bayley MDI & 77 & -1.20 & 13.00 & 77 & -1.20 & 13.00 \\
\hline Morley (39) & Bayley MDI & 133 & 0.20 & 11.59 & 135 & 0.50 & 11.59 \\
\hline \multicolumn{8}{|l|}{ Language } \\
\hline Soemantri 1 (36) & Language & 37 & -2.27 & 6.98 & 35 & 1.48 & 6.98 \\
\hline Soemantri 2 (36) & Language & 34 & 12.55 & 3.52 & 24 & 4.62 & 3.52 \\
\hline Pollitt 1 (15) & Language & 50 & 5.85 & 4.31 & 51 & 5.96 & 4.31 \\
\hline Pollitt 2 (15) & Language & 24 & 1.48 & 7.39 & 23 & 0.96 & 7.39 \\
\hline Pollitt 3 (15) & Language & 605 & 6.30 & 1.92 & 605 & 4.50 & 1.92 \\
\hline Stoltzfus (40) & Language & 183 & 3.70 & 5.80 & 176 & 3.70 & 5.80 \\
\hline \multicolumn{8}{|l|}{ Mathematics } \\
\hline Soemantri 1 (36) & Mathematics & 37 & 4.48 & 4.22 & 35 & 4.50 & 4.22 \\
\hline Soemantri 2 (36) & Mathematics & 34 & 9.40 & 3.24 & 24 & 1.30 & 3.24 \\
\hline Pollitt 1 (15) & Mathematics & 50 & 0.00 & 3.73 & 51 & 7.91 & 3.73 \\
\hline Pollitt 2 (15) & Mathematics & 24 & 4.16 & 7.79 & 23 & 7.50 & 7.79 \\
\hline Pollitt 3 (15) & Mathematics & 605 & 5.25 & 2.12 & 605 & 5.80 & 2.12 \\
\hline \multicolumn{8}{|l|}{ Visual recall } \\
\hline Seshadri Ca (21) & Visual recall & 16 & 1.65 & 1.28 & 8 & 0.10 & 1.28 \\
\hline Seshadri Cb (21) & Visual recall & 16 & 1.38 & 1.61 & 8 & 0.10 & 1.61 \\
\hline Seshadri D1 (21) & Visual recall & 36 & 0.56 & 2.91 & 45 & 0.76 & 2.91 \\
\hline Seshadri D2 (21) & Visual recall & 10 & 0.70 & 1.36 & 10 & 0.75 & 1.36 \\
\hline \multicolumn{8}{|l|}{ Digit span } \\
\hline Seshadri Ca (21) & Digit span & 16 & 0.81 & 1.81 & 8 & 0.10 & 1.81 \\
\hline Seshadri Cb (21) & Digit span & 16 & 1.16 & 1.73 & 8 & 0.10 & 1.73 \\
\hline Seshadri D1 (21) & Digit span & 36 & 0.88 & 1.52 & 45 & 0.42 & 1.52 \\
\hline Seshadri D2 (21) & Digit span & 10 & 1.32 & 1.07 & 10 & 0.37 & 1.07 \\
\hline \multicolumn{8}{|l|}{ Mazes } \\
\hline Seshadri Ca (21) & Mazes & 16 & 2.88 & 3.61 & 8 & 0.09 & 3.61 \\
\hline Seshadri Cb (21) & Mazes & 16 & 4.82 & 3.89 & 8 & 0.09 & 3.89 \\
\hline Seshadri D1 (21) & Mazes & 36 & 6.00 & 5.74 & 45 & 3.20 & 5.74 \\
\hline Seshadri D2 (21) & Mazes & 10 & 5.70 & 3.36 & 10 & 3.35 & 3.36 \\
\hline \multicolumn{8}{|l|}{ Clerical tasks } \\
\hline Seshadri Ca (21) & Clerical tasks & 16 & 0.91 & 1.55 & 8 & 0.11 & 1.55 \\
\hline Seshadri Cb (21) & Clerical tasks & 16 & 0.84 & 1.33 & 8 & 0.11 & 1.33 \\
\hline Seshadri D1 (21) & Clerical tasks & 36 & 1.93 & 1.84 & 45 & 0.77 & 1.84 \\
\hline Seshadri D2 (21) & Clerical tasks & 10 & 1.97 & 1.51 & 10 & 0.87 & 1.51 \\
\hline \multicolumn{8}{|l|}{ Motor development scale } \\
\hline Kimmons (unpublished) & Bayley PDI & 17 & 0.20 & 9.73 & 17 & 3.30 & 9.73 \\
\hline
\end{tabular}


Table 2 Continued

\begin{tabular}{|c|c|c|c|c|c|c|c|}
\hline \multirow[b]{2}{*}{ Author (reference) } & \multirow[b]{2}{*}{ Outcome } & \multicolumn{3}{|c|}{$\begin{array}{l}\text { Change in iron } \\
\text { supplement group }\end{array}$} & \multicolumn{3}{|c|}{$\begin{array}{l}\text { Change in } \\
\text { placebo group }\end{array}$} \\
\hline & & Number & Mean & SD & Number & Mean & SD \\
\hline Oski (13) & Bayley PDI & 12 & 11.00 & 21.22 & 12 & 4.17 & 21.22 \\
\hline Lozoff 1 (30) & Bayley PDI & 12 & 0.50 & 7.40 & 12 & 5.70 & 7.40 \\
\hline Lozoff $2(30)$ & Bayley PDI & 17 & -1.60 & 7.40 & 18 & 1.30 & 7.40 \\
\hline Driva (31) & Bayley PDI & 20 & 0.80 & 9.50 & 20 & 3.50 & 9.50 \\
\hline Aukett (33) & DDST & 48 & 4.00 & 2.60 & 49 & 3.20 & 2.30 \\
\hline Walter 1 (35) & Bayley PDI & 24 & 6.70 & 6.90 & 15 & 5.10 & 2.90 \\
\hline Walter 2 (35) & Bayley PDI & 66 & 5.60 & 2.90 & 61 & 5.40 & 3.50 \\
\hline Walter 3 (35) & Bayley PDI & 12 & 5.60 & 3.20 & 18 & 4.40 & 4.30 \\
\hline Idjradinata 1 (37) & Bayley PDI & 24 & 23.50 & 14.27 & 23 & 5.10 & 14.27 \\
\hline Idjradinata 2 (37) & Bayley PDI & 14 & 4.90 & 14.27 & 14 & 3.10 & 14.27 \\
\hline Idjradinata 3 (37) & Bayley PDI & 22 & 3.40 & 14.27 & 22 & 2.40 & 14.27 \\
\hline Mofatt (38) & Bayley PDI & 77 & -2.10 & 12.63 & 77 & -4.10 & 12.63 \\
\hline Morley (39) & Bayley PDI & 133 & -0.10 & 8.96 & 135 & 0.00 & 8.96 \\
\hline Stoltzfus (40) & Motor score & 132 & 4.70 & 5.03 & 123 & 4.60 & 5.03 \\
\hline
\end{tabular}

MDI - Mental Development Index; IQ - intelligence quotient; PPVT - Peabody Picture Vocabulary Test; PDI - Psychomotor Development Index; DDST - Denver Development Screening Test.

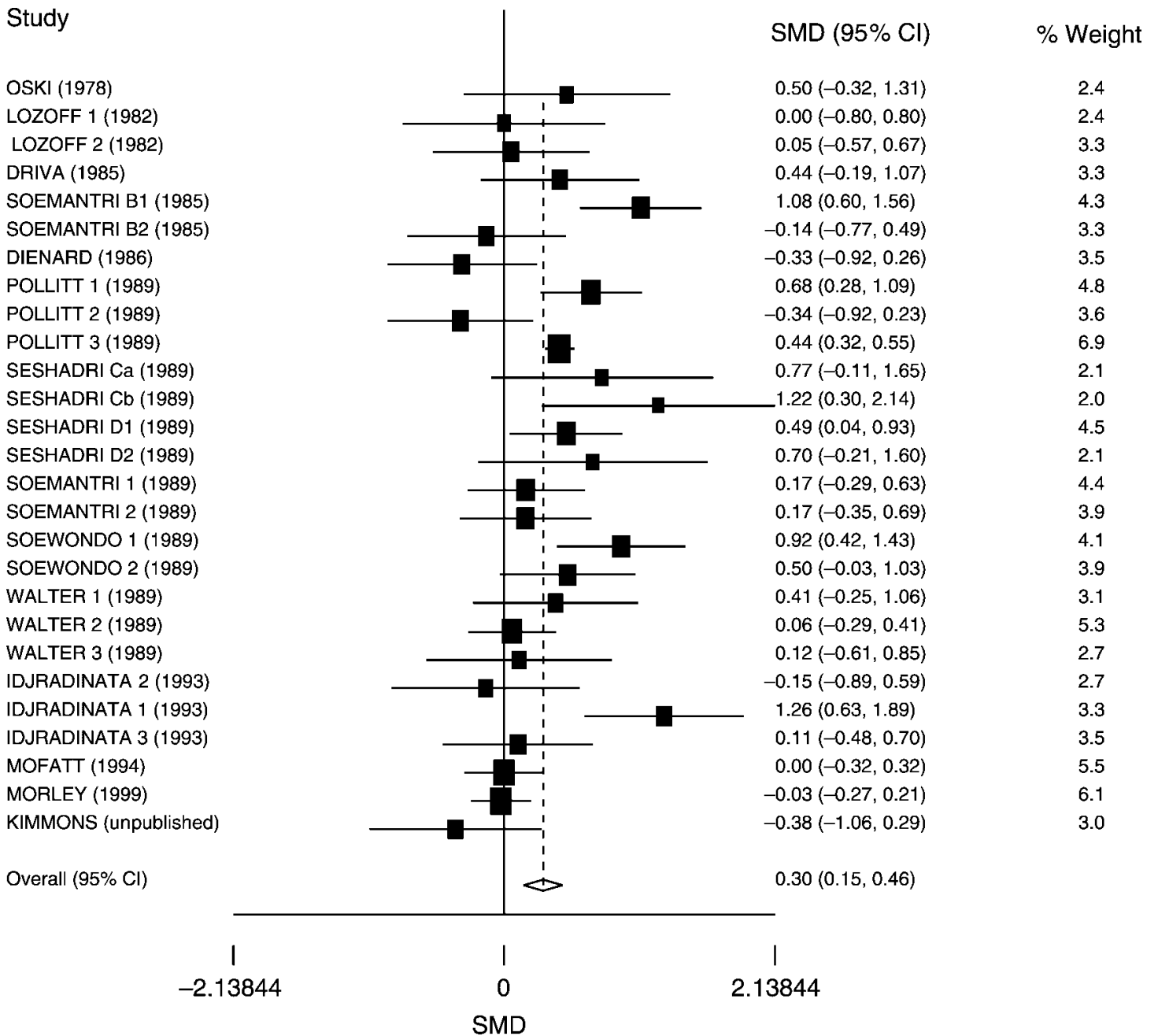

Fig. 3 Forest plot for mental development score with unknown standard deviations derived under the assumption $P=0.5$. SMD - standardised mean difference; $\mathrm{Cl}$ - confidence interval 
Table 3 Sensitivity analyses of pooled estimates of mental development score standardised mean difference

\begin{tabular}{|c|c|c|c|c|}
\hline Stratification variable & $\begin{array}{l}\text { No. of analytic } \\
\text { components }\end{array}$ & $\begin{array}{l}\text { Random effects } \\
\text { model }(95 \% \mathrm{Cl})\end{array}$ & $P$-value & $\begin{array}{l}\text { Test for heterogeneity } \\
(P \text {-value })\end{array}$ \\
\hline \multicolumn{5}{|l|}{ All } \\
\hline SD by $P=0.5$ & 27 & $0.30(0.15,0.46)$ & $<0.001$ & $72.05(<0.001)$ \\
\hline SD by independence & 27 & $0.25(0.12,0.38)$ & $<0.001$ & $51.65(0.002)$ \\
\hline Post-test score and SD & 27 & $0.19(0.04,0.34)$ & 0.013 & $68.74(<0.001)$ \\
\hline \multicolumn{5}{|l|}{ Supplementation route } \\
\hline Fortification & 2 & $-0.02(-0.21,0.17)$ & 0.866 & $0.02(0.898)$ \\
\hline Oral & 22 & $0.36(0.20,0.53)$ & $<0.001$ & $52.10(<0.001)$ \\
\hline Parenteral & 3 & $0.17(-0.39,0.74)$ & 0.551 & $3.9(0.142)$ \\
\hline \multicolumn{5}{|l|}{ Duration of supplementation } \\
\hline$<1$ month & 8 & $0.12(-0.08,0.33)$ & 0.243 & $4.93(0.669)$ \\
\hline$>1$ month & 19 & $0.36(0.17,0.55)$ & $<0.001$ & $62.97(<0.001)$ \\
\hline \multicolumn{5}{|l|}{ Mean age } \\
\hline$<24$ months & 13 & $0.15(-0.04,0.34)$ & 0.128 & $19.98(0.067)$ \\
\hline$<60$ months & 16 & $0.21(0.01,0.41)$ & 0.043 & $33.77(0.004)$ \\
\hline$>60$ months & 11 & $0.44(0.21,0.66)$ & $<0.001$ & $24.65(0.006)$ \\
\hline \multicolumn{5}{|l|}{ Allocation concealment } \\
\hline Adequate & 3 & $-0.04(-0.23,0.14)$ & 0.643 & $1.06(0.588)$ \\
\hline Others & 24 & $0.37(0.21,0.53)$ & $<0.001$ & $52.17(<0.001)$ \\
\hline \multicolumn{5}{|l|}{ Attrition } \\
\hline$<10 \%$ & 17 & $0.37(-0.15,0.59)$ & $<0.001$ & $36.41(0.003)$ \\
\hline$>10 \%$ & 10 & $0.22(-0.02,0.46)$ & 0.069 & $35.58(<0.001)$ \\
\hline \multicolumn{5}{|l|}{ Blinding } \\
\hline Double blind & 15 & $0.36(0.19,0.53)$ & $<0.001$ & $29.16(0.010)$ \\
\hline Others & 12 & $0.21(-0.09,0.51)$ & 0.178 & $36.72(<0.001)$ \\
\hline \multicolumn{5}{|l|}{ Mean baseline $\mathrm{Hb}$} \\
\hline$<11 \mathrm{~g} \mathrm{dl}^{-1}$ & 14 & $0.49(0.23,0.74)$ & $<0.001$ & $39.82(<0.001)$ \\
\hline$>11 \mathrm{~g} \mathrm{dl}^{-1}$ & 13 & $0.14(-0.06,0.34)$ & 0.181 & $31.43(0.002)$ \\
\hline \multicolumn{5}{|l|}{ Iron deficiency status } \\
\hline Deficient, anaemic & 11 & $0.50(0.25,0.75)$ & $<0.001$ & $21.57(0.017)$ \\
\hline Deficient, non-anaemic & 4 & $-0.11(-0.36,0.14)$ & 0.386 & $2.12(0.548)$ \\
\hline Deficient, \pm anaemic & 15 & $0.31(0.06,0.56)$ & 0.014 & $41.34(<0.001)$ \\
\hline Replete & 8 & $0.33(0.11,0.55)$ & 0.003 & $11.40(0.122)$ \\
\hline
\end{tabular}

$\mathrm{Cl}$ - confidence interval; SD - standard deviation; $\mathrm{Hb}$ - haemoglobin.

Except the all category, these calculations were performed with SD calculated under the assumption $P=0.5$.

Table 4 Meta-regression analyses for mental development score standardised mean difference (SMD)

\begin{tabular}{|c|c|c|c|c|}
\hline \multirow[b]{2}{*}{ Study characteristic } & \multicolumn{2}{|c|}{ Univariable analysis } & \multicolumn{2}{|c|}{ Controlling for all variables } \\
\hline & SMD (95\% Cl) & $P$-value & SMD $(95 \% \mathrm{Cl})$ & $P$-value \\
\hline \multicolumn{5}{|l|}{ Study quality } \\
\hline Allocation concealment (not adequate vs. adequate) & $0.46(0.03,0.89)$ & 0.035 & $0.53(-0.17,1.22)$ & 0.136 \\
\hline Attrition ( $>10 \%$ vs. $<10 \%$ ) & $-0.16(-0.49,0.18)$ & 0.367 & $0.06(-0.29,0.41)$ & 0.742 \\
\hline Blinding (not double blind vs. double blind) & $-0.17(-0.50,0.17)$ & 0.333 & $-0.12(-0.46,0.22)$ & 0.478 \\
\hline Unit increase in duration of iron supplementation (months) & $-0.01(-0.07,0.05)$ & 0.797 & $0.01(-0.06,0.08)$ & 0.775 \\
\hline Unit increase in mean age (months) & $0.00(-0.00,0.01)$ & 0.060 & $0.00(-0.00,0.00)$ & 0.536 \\
\hline Unit increase in mean baseline $\mathrm{Hb}$ status $\left(\mathrm{g} \mathrm{dl}^{-1}\right)$ & $-0.11(-0.23,0.01)$ & 0.069 & $-0.14(-0.03,-0.25)$ & 0.012 \\
\hline Iron deficiency status* (deficient, anaemic vs. others) & $0.33(0.02,0.64)$ & 0.036 & $0.30(0.09,0.51)$ & 0.005 \\
\hline
\end{tabular}

$\mathrm{Cl}$ - confidence interval; $\mathrm{Hb}$ - haemoglobin.

${ }^{*}$ For the multivariable model, mean baseline $\mathrm{Hb}$ status was replaced by a dichotomous variable (deficient and anaemic $=1$, others including unknown status $=0)$.

the pooled IQ scores. There was no effect of iron supplementation on motor development.

Our conclusion that mental development scores improved following iron supplementation was based on a large spectrum of sensitivity analyses. Significant explanatory variables could be identified to explain heterogeneity, specifically initial iron status and $\mathrm{Hb}$. None of the analyses showed evidence of publication bias, and omitting one study at a time (data not shown) did not reveal an overwhelming effect of any study.
Seven limitations merit consideration. First, the review attempted to combine all studies examining mental or motor development irrespective of age, instrument used, setting or the specific aspect of development evaluated. However, the majority of the mental development scores, including Bayley MDI, Stanford Binet score, IQ and cognition score, assess general intelligence or overall mental development in children. Hence, we believe that combining these studies is a logical summary of the mental development effect for guiding 
Table 5 Pooled estimates of individual mental and motor development tests

\begin{tabular}{|c|c|c|c|c|}
\hline Stratification variable & $\begin{array}{l}\text { No. of analytic } \\
\text { components }\end{array}$ & $\begin{array}{l}\text { Random effects } \\
\text { model }(95 \% \mathrm{Cl})\end{array}$ & $P$-value & $\begin{array}{l}\text { Tests for heterogeneity } \\
\text { ( } P \text {-value })\end{array}$ \\
\hline \multicolumn{5}{|l|}{ Bayley MDI (WMD) } \\
\hline SD by $P=0.5$ & 13 & $0.95(-0.56,2.46)$ & 0.217 & $24.72(0.016)$ \\
\hline SD by independence & 13 & $1.06(-0.54,2.66)$ & 0.196 & $24.56(0.017)$ \\
\hline Post-test scores and SD & 13 & $1.67(-1.14,4.49)$ & 0.244 & $32.48(0.001)$ \\
\hline \multicolumn{5}{|c|}{ Bayley MDI and Stanford Binet test score (SMD) } \\
\hline SD by $P=0.5$ & 14 & $0.12(-0.07,0.30)$ & 0.219 & $21.94(0.056)$ \\
\hline SD by independence & 14 & $0.12(-0.06,0.30)$ & 0.187 & $21.05(0.072)$ \\
\hline Post-test scores and SD & 14 & $0.13(-0.08,0.35)$ & 0.221 & $28.78(0.007)$ \\
\hline \multicolumn{5}{|l|}{ IQ (SMD) } \\
\hline SD by $P=0.5$ & 9 & $0.41(0.20,0.62)$ & $<0.001$ & $14.43(0.071)$ \\
\hline SD by independence & 9 & $0.30(0.20,0.40)$ & $<0.001$ & $7.40(0.494)$ \\
\hline Post-test scores and SD & 9 & $0.25(-0.07,0.57)$ & 0.122 & $34.36(<0.001)$ \\
\hline \multicolumn{5}{|l|}{ Digit span (WMD) } \\
\hline SD by $P=0.5$ & 4 & $0.68(0.20,1.16)$ & 0.006 & $0.99(0.803)$ \\
\hline SD by independence & 4 & $0.69(-0.01,1.38)$ & 0.052 & $0.49(0.922)$ \\
\hline Post-test scores and SD & 4 & $0.37(-0.24,0.97)$ & 0.234 & $4.52(0.211)$ \\
\hline \multicolumn{5}{|l|}{ Visual recall (WMD) } \\
\hline SD by $P=0.5$ & 4 & $0.66(-0.24,1.56)$ & 0.152 & $6.46(0.091)$ \\
\hline SD by independence & 4 & $0.74(-0.15,1.62)$ & 0.103 & $3.54(0.315)$ \\
\hline Post-test scores and SD & 4 & $0.88(0.07,1.68)$ & 0.033 & $7.15(0.067)$ \\
\hline \multicolumn{5}{|l|}{ Mazes (WMD) } \\
\hline SD by $P=0.5$ & 4 & $3.06(1.61,4.52)$ & $<0.001$ & $1.28(0.734)$ \\
\hline SD by independence & 4 & $3.17(1.27,5.06)$ & 0.001 & $0.85(0.837)$ \\
\hline Post-test scores and SD & 4 & $1.24(0.61,1.88)$ & $<0.001$ & $0.36(0.948)$ \\
\hline \multicolumn{5}{|l|}{ Clerical task (WMD) } \\
\hline SD by $P=0.5$ & 4 & $0.99(0.46,1.53)$ & $<0.001$ & $0.48(0.923)$ \\
\hline SD by independence & 4 & $0.96(0.24,1.68)$ & 0.009 & $0.28(0.964)$ \\
\hline Post-test scores and SD & 4 & $0.34(-0.31,0.99)$ & 0.302 & $4.64(0.200)$ \\
\hline \multicolumn{5}{|l|}{ Language score (SMD) } \\
\hline SD by $P=0.5$ & 6 & $0.42(-0.18,1.02)$ & 0.166 & $121.86(<0.001)$ \\
\hline SD by independence & 6 & $0.30(-0.14,0.74)$ & 0.180 & $64.52(<0.001)$ \\
\hline Post-test scores and SD & 6 & $0.57(-0.10,1.23)$ & 0.096 & $146.34(<0.001)$ \\
\hline \multicolumn{5}{|l|}{ Mathematics score (WMD) } \\
\hline SD by $P=0.5$ & 5 & $-0.67(-5.04,3.69)$ & 0.762 & $200.31(<0.001)$ \\
\hline SD by independence & 5 & $-0.62(-5.04,3.79)$ & 0.782 & $99.89(<0.001)$ \\
\hline Post-test scores and SD & 5 & $-0.16(-2.76,2.44)$ & 0.905 & $81.98(<0.001)$ \\
\hline \multicolumn{5}{|l|}{ Bayley PDI (WMD) } \\
\hline SD by $P=0.5$ & 13 & $0.52(-1.22,2.27)$ & 0.558 & $28.50(0.005)$ \\
\hline SD by independence & 13 & $0.51(-1.36,2.38)$ & 0.595 & $28.10(0.005)$ \\
\hline Post-test scores and SD & 13 & $1.47(-1.35,4.29)$ & 0.308 & $33.28(0.001)$ \\
\hline \multicolumn{5}{|l|}{ Bayley PDI and DDST (SMD) } \\
\hline SD by $P=0.5$ & 14 & $0.10(-0.09,0.30)$ & 0.297 & $25.40(0.020)$ \\
\hline SD by independence & 14 & $0.10(-0.10,0.29)$ & 0.317 & $25.21(0.022)$ \\
\hline Post-test scores and SD & 14 & $0.11(-0.12,0.34)$ & 0.341 & $28.62(0.004)$ \\
\hline
\end{tabular}

$\mathrm{Cl}$-confidence interval; MDI - Mental Development Index; WMD - weighted mean difference; SD - standard deviation; SMD - standardised mean difference; IQ - intelligence quotient; PDI - Psychomotor Development Index; DDST - Denver Development Screening Test.

policy. A similar logic was used for motor development. To retain purity in the evaluated outcomes, we also conducted separate stratified analyses for specific individual tests.

Second, the sensitivities of the mental and motor development tests were different and this variability could not be quantified in the analytical process. DDST was used to assess the psychomotor development of children ${ }^{33}$. This test is a screening tool for single-time assessment of development; it is not designed to assess psychomotor development or the change in psychomotor development over a period of time with accuracy or a high degree of sensitivity. The IQ scales used in older children may have been more sensitive, and may explain the different results across the different age groups. Nevertheless, the contribution of this factor to differences in pooled estimates is speculative.

Third, most of the included trials did not control for differences in socio-economic status and the extent of stimulation provided to the children. This is important, because lower cognition scores in iron-deficient children have often been attributed to other confounding environmental factors such as poverty, lack of stimulation, undernutrition, maternal factors and worm infestation ${ }^{5-7}$. Because the trials included were randomised and controlled, most of these factors would have been controlled for.

Fourth, we could not confidently differentiate the therapeutic from the preventive effects of iron supplementation as few studies provided relevant data or were 


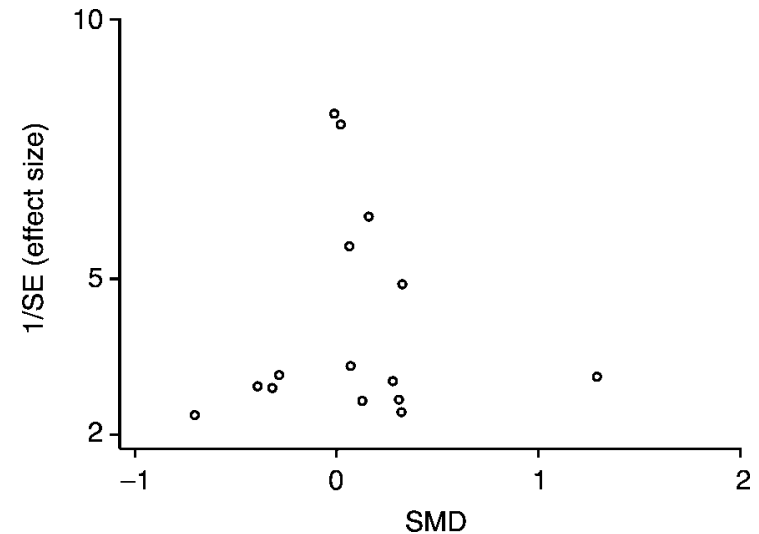

Fig. 4 Funnel plot for motor development scores with unknown standard deviations derived under the assumption $P=0.5$. SE standard error; SMD - standardised mean difference

designed as preventive interventions. An approximation of the preventive or therapeutic role can be inferred by relating the outcome to iron status.

Fifth, it was assumed that all cases of anaemia were attributable to iron deficiency. Iron deficiency is usually the most common cause of anaemia in childhood ${ }^{41}$, but its contribution is variable in different countries depending upon the prevalence of hookworm infestation and malaria ${ }^{42}$. Among the studies included, only 10 determined the iron status of the children.

Sixth, the iron supplement dose could not be directly related to the observed effect because the majority of the trials did not provide these data. Thus, we assumed that the fortification trials used the lowest dose, parenteral studies the maximum dose, and oral iron supplementation studies a level in between.

Finally, in the absence of actual data on the variability of the change in outcome scores, several imputations were made based on pre-specified assumptions. The sensitivity analyses suggested that these imputations were robust because the quantification of the findings with various assumptions was invariably synchronous.

Like an earlier systematic review restricted to children with IDA below 3 years of age ${ }^{7}$, we found no evidence of a beneficial effect of iron supplementation on motor development, even in iron-deficient and anaemic children. Nevertheless, because of the relatively small number of children included in these studies, the confidence intervals around the effects of treatment are wide and the results could be compatible with moderate positive or adverse effects of short-term iron therapy ${ }^{7}$. Three other possible explanations exist for this finding. First, iron deficiency may cause irreversible structural brain changes, particularly in younger children. Evidence from animal studies provides support of this possibility $^{1-4}$. Second, the tests evaluated (for example, Bayley PDI) may not be sufficiently sensitive measures of motor development, particularly in younger subjects. Third, the duration of iron supplementation in several trials may have been too short to correct the iron deficiency (8/13 studies evaluating Bayley indices intervened for less than 1 month).

An approximation of the effect size of the mental development score can be derived by relating the

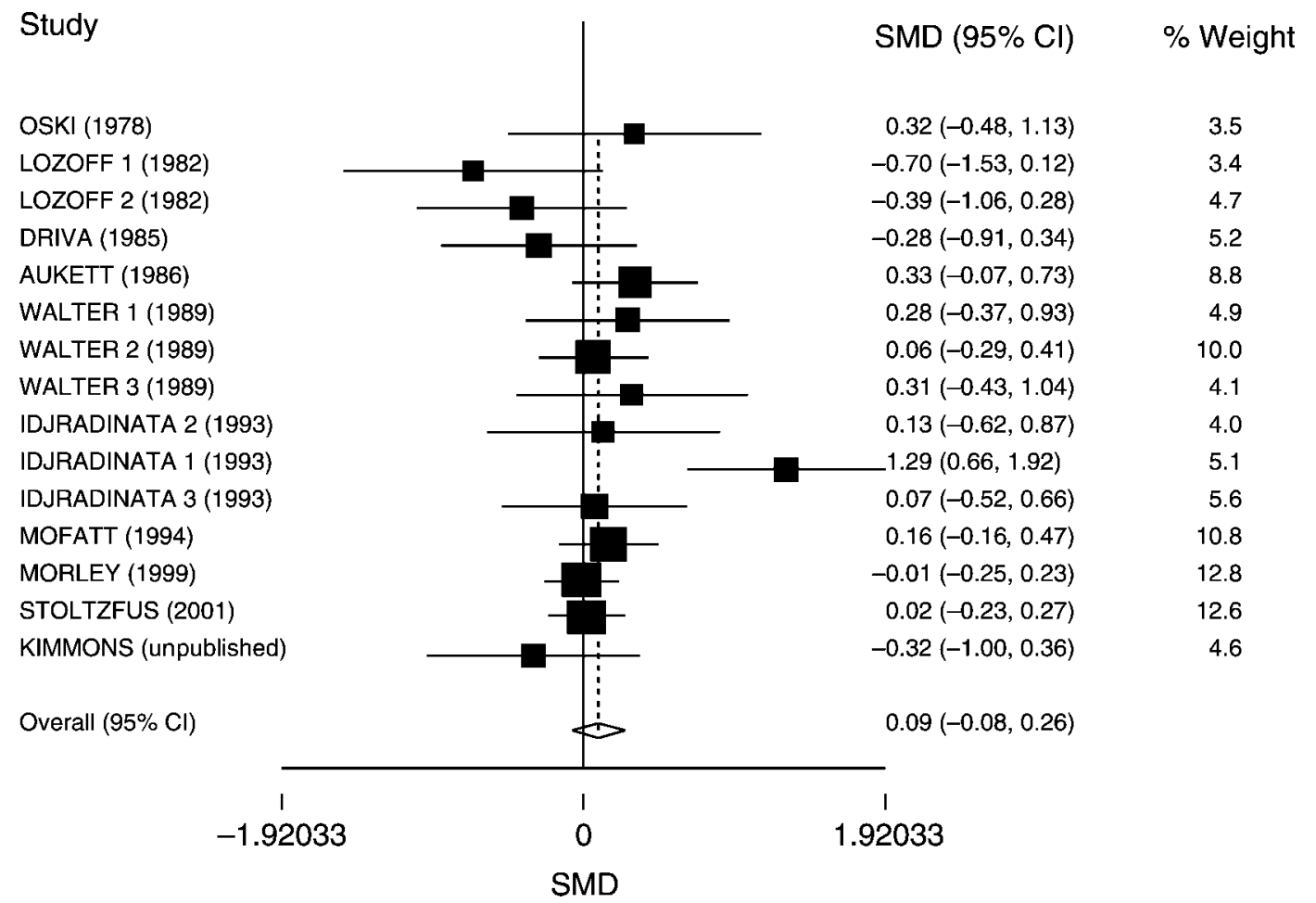

Fig. 5 Forest plot for motor development score with unknown standard deviations derived under the assumption $P=0.5$. SMD - standardised mean difference; $\mathrm{Cl}$ - confidence interval 
Table 6 Meta-regression analyses for motor development score standardised mean difference (SMD)

\begin{tabular}{|c|c|c|c|c|}
\hline \multirow[b]{2}{*}{ Study characteristic } & \multicolumn{2}{|c|}{ Univariable analysis } & \multicolumn{2}{|c|}{ Controlling for all variables } \\
\hline & $\operatorname{SMD}(95 \% \mathrm{Cl})$ & $P$-value & $\operatorname{SMD}(95 \% \mathrm{Cl})$ & $P$-value \\
\hline \multicolumn{5}{|l|}{ Study quality } \\
\hline Allocation concealment (not adequate vs. adequate) & $0.06(-0.29,0.41)$ & 0.745 & $-0.32(-1.31,0.65)$ & 0.519 \\
\hline Attrition ( $>10 \%$ vs. $<10 \%)$ & $-0.26(-0.59,0.08)$ & 0.132 & $-0.69(-1.47,0.08)$ & 0.080 \\
\hline Blinding (not double blind vs. double blind) & $-0.13(-0.47,0.21)$ & 0.448 & $-0.10(-0.56,0.35)$ & 0.661 \\
\hline Unit increase in duration of iron supplementation (months) & $0.01(-0.03,0.05)$ & 0.769 & $0.02(-0.05,0.09)$ & 0.607 \\
\hline Unit increase in mean age (months) & $-0.00(-0.03,0.02)$ & 0.854 & $-0.01(-0.04,0.03)$ & 0.698 \\
\hline Unit increase in mean baseline $\mathrm{Hb}$ status $\left(\mathrm{g} \mathrm{dl}^{-1}\right)$ & $-0.02(-0.15,0.10)$ & 0.719 & $-0.05(-0.24,0.13)$ & 0.568 \\
\hline Iron deficiency status* (deficient, anaemic vs. others) & $0.09(-0.25,0.43)$ & 0.585 & $0.27(-0.27,0.82)$ & 0.327 \\
\hline
\end{tabular}

$\mathrm{Cl}$ - confidence interval; $\mathrm{Hb}-$ haemoglobin.

${ }^{*}$ For the multivariable model, mean baseline $\mathrm{Hb}$ status was replaced by a dichotomous variable (deficient and anaemic $=1$, others including unknown status $=0)$.

standardised mean differences to actual mean differences whenever feasible. Thus, for Bayley MDI, a standardised mean difference of 0.147 was equivalent to a mean difference of 0.949 (a conversion factor of 6.5). Similarly, for IQ scores a standardised mean difference of 0.41 was equivalent to a mean difference of 1.96 (a conversion factor of 5). Extrapolating to mental development score on a scale of 100 , a reasonable estimate of standardised mean difference of 0.30 would be between 1.5 to 2 points, which is 'modest'.

A significant improvement was evident in intelligence tests conducted in children over 7 years old, whereas no benefit was documented in Bayley MDI amongst those below 27 months of age. These differences could be real. However, another review ${ }^{43}$ has attributed this differential benefit to better designed studies, increased sensitivity of the instruments used and the possibility of a transitory effect of iron deficiency on these tests. Animal studies indicate that adverse effects of iron deficiency on neurotransmitter systems, such as monoamine oxidase, can be reversed by supplementation ${ }^{44}$. Conversely, lack of benefit in Bayley MDI scores could reflect irreversible effects of iron deficiency on rapidly developing brain. In animal studies, dietary iron deficiency during the period of maximal brain growth leads to irreversible effects ${ }^{3,4,45-47}$.

The improvement in intelligence scores in older children, and particularly in those who were iron-deficient and anaemic, suggests a causal role of iron in mental development. Furthermore, the reversibility of the cognitive deficit, even if partial and restricted to a subset, lends support to advocacy for public health programmes to control iron deficiency.

The documentation of a significant benefit in mental development score in iron-sufficient children suggests a possible preventive role. Suitably designed trials are required to critically evaluate the role of preventive supplementation, particularly in younger children. It has been hypothesised that iron supplementation could benefit specific components of mental development with no demonstrable evidence on the total score ${ }^{29,40}$. We cannot address this issue from the available data.

\section{Conclusion}

Most observational studies have found associations between IDA and poor mental and motor development in children. Conflicting data exist regarding the possibility of improved mental and motor development with iron administration, resulting in confusion about realistic expectations from iron supplementation and fortification efforts.

Our meta-analysis indicates that iron supplementation improves mental development score, but the effect is modest (SMD of 0.3 , equivalent to 1.5 to 2 points on a scale of 100). This effect is particularly apparent for intelligence tests above 7 years of age, and in initially anaemic or irondeficient anaemic subjects. There is no convincing evidence that iron treatment has an effect on mental development in children below 27 months of age, or on motor development.

\section{Acknowledgements}

We thank Clive Osmond for offering helpful advice for statistical analysis.

Contributors: T.G. prepared the protocol, applied the search strategy, performed the retrieval of articles and extracted the data from the studies. H.P.S.S. and P.N. developed the idea for the review, finalised the protocol and search strategy. H.P.S.S. performed the statistical analysis. All authors contributed to the drafting of the final version of the paper. H.P.S.S. and T.G. are the guarantors.

Funding: The work was supported by The United States Agency for International Development through its co-operative agreement (No. HRN-A-00-98-00027-00) with the Human Nutrition Institute of the International Life Sciences Institute (ILSI) Research Foundation. The funding source had no influence on the study design, analysis and interpretation, and the decision to submit for publication.

Competing interests: H.P.S.S. is an honorary member of the International Nutritional Anaemia Consultative Group (INACG) Steering Committee that is managed by 
ILSI. T.G. was supported by ILSI for travel to Hanoi, Vietnam and Marrakech, Morocco for presenting research work in the annual INACG symposiums. P.N. was a consultant to ILSI.

\section{References}

1 Yehuda S, Youdim MBH, Mostofsky DI. Brain iron deficiency causes reduced learning capacity in rats. Pharmacology, Biochemistry, and Behavior 1986; 25: 141-4.

2 Beard JL, Connor JR, Jones BC. Brain iron: location and function. Progress in Food \& Nutrition Science 1993; 17: 183-221.

3 Beard JL, Connor JR, Jones BC. Iron in the brain. Nutrition Reviews 1993; 51: 157-70.

4 Connor JR, Menzies SL. Relationship of iron to oligodendrocytes and myelination. Glia 1996; 17: 83-93.

5 Lansdown R, Wharton BA. Iron and mental and motor behaviour in children. In: Iron, Nutrition and Physiological Significance: Report of the British Nutrition Task Force. London: Chapman and Hall, 1995; 65-78.

6 Grantham-McGregor S, Ani C. A review of studies on the effect of iron deficiency on cognitive development in children. Journal of Nutrition 2001; 131: 649S-68S.

7 Logan S, Martins S, Gilbert R. Iron therapy for improving psychomotor development and cognitive function in children under the age of three with iron deficiency anaemia (Cochrane Review). In: The Cochrane Library, Issue 4. Oxford: Update Software, 2001.

8 Juni $\mathrm{P}$, Altman DG, Egger M. Assessing the quality of randomised controlled trials. In: Egger M, Smith GD, Altman DG, eds. Systematic Reviews in Health Care: Metaanalysis in Context. London: BMJ Publishing, 2001; 87-108.

9 Clarke M, Oxman AD, eds. Assessment of study quality. Cochrane Reviewers Handbook 4.1.1, updated December 2000. In: The Cochrane Library, Issue 1. Oxford: Update Software, 2001.

10 Follmann D, Elliott P, Suh I, Cutler J. Variance imputation for overviews of clinical trials with continuous response. Journal of Clinical Epidemiology 1992; 45: 769-73.

11 Sterne JAC, Egger M, Smith GD. Investigating and dealing with publication and other biases. In: Egger M, Smith GD, Altman DG, eds. Systematic Reviews in Health Care: Metaanalysis in Context. London: BMJ Publishing, 2001; 189-208.

12 Sterne JAC, Bradburn MJ, Egger M. Meta-analysis in STATA ${ }^{\mathrm{TM}}$. In: Egger M, Smith GD, Altman DG, eds. Systematic Reviews in Health Care: Meta-analysis in Context. London: BMJ Publishing, 2001; 347-69.

13 Oski FA, Honig AS. The effects of therapy on the developmental scores of iron-deficient infants. Journal of Pediatrics 1978; 92: 21-5.

14 Dienard AS, List A, Lindgren B, Hunt JV, Chang PN. Cognitive deficits in iron-deficient and iron deficient anemic children. Journal of Pediatrics 1986; 108: 681-9.

15 Pollitt E, Hathirat P, Kotchabhakdi NJ, Missell L, Valyasevi A. Iron deficiency and educational achievement in Thailand. American Journal of Clinical Nutrition 1989; 50: 687-97.

16 Cantwell RJ. The long-term neurological sequelae of anaemia in infancy. Pediatric Research 1974; 342: 68.

17 Bruner AB, Joffe A, Duggan AK, Casella JF, Brandt J. Randomised study of cognitive effects of iron supplementation in non-anaemic iron-deficient adolescent girls. Lancet 1996; 348: 992-6.

18 Oski FA, Honig AS, Helu B, Howanitz P. Effect of iron therapy on behavior performance in nonanemic, irondeficient infants. Pediatrics 1983; 71: 877-80.
19 Pollitt E, Soemantri AG, Yunis F, Scrimshaw NS. Cognitive effects of iron deficiency anaemia. Lancet 1985; 1: 158.

20 Lozoff B, Brittenham GM, Wolf AW, McClish DK, Kuhnert PM, Jimenez R, et al. Iron deficiency anemia and iron therapy effects on infant developmental test performance. Pediatrics 1987; 79: 981-95.

21 Seshadri S, Gopaldas T. Impact of iron supplementation on cognitive functions in preschool and school-aged children: the Indian experience. American Journal of Clinical Nutrition 1989; 50(Suppl. 3): 675-84.

22 Heywood A, Oppenheimer S, Heywood P, Jolley D. Behavioral effects of iron supplementation in infants in Madang, Papua New Guinea. American Journal of Clinical Nutrition 1989; 50(Suppl. 3): 630-7.

23 Boivin MJ, Giordani B. Improvements in cognitive performance for school children in Zaire, Africa, following an iron supplement and treatment for intestinal parasites. Journal of Pediatric Psychology 1993; 18: 249-64.

24 Lozoff B, Wolf AW, Jimenez E. Iron deficiency anemia and infant development: effects of extended oral iron therapy. Journal of Pediatrics 1996; 129: 382-9.

25 Lynn R, Harland P. A positive effect of iron supplementation on the IQs of iron deficient children. Personality and Individual Differences 1998; 24: 883-5.

26 Williams J, Wolff A, Daly A, Macdonald A, Aukett A, Booth AW. Iron supplemented formula milk related to reduction in psychomotor decline in infants from inner city areas: randomised study. British Medical Journal 1999; 318 : $693-7$.

27 Walka H, Triana N, Jahari AB, Husaini AM, Pollitt E. Effects of an energy and micronutrient supplement on play behaviour in undernourished children in Indonesia. European Journal of Clinical Nutrition 2000; 54(Suppl. 2): S91-106.

28 Pollitt E, Jahari A, Walka H. A developmental view of the effects of an energy and micronutrient supplement in undernourished children in Indonesia. European Journal of Clinical Nutrition 2000; 54(Suppl. 2): S107-13.

29 Lozoff B, De Andraca I, Castillo M, Smith JB, Walter T, Pino P. Behavioral and developmental effects of preventing iron deficiency anemia in healthy full-term infants. Pediatrics 2003; 112: 846-54.

30 Lozoff B, Brittenham GM, Viteri FE, Wolf AW, Urrutia JJ. The effects of short-term oral iron therapy on developmental deficits in iron deficient anemic infants. Journal of Pediatrics 1982; 100: 351-7.

31 Driva A, Kafatos A, Salman M. Iron deficiency and the cognitive and psychomotor development of children: a pilot study with institutionalised children. Early Child Development and Care 1985; 22: 73-82.

32 Soemantri AG, Pollitt E, Kim I. Iron deficiency anemia and educational achievement. American Journal of Clinical Nutrition 1985; 42: 1221-8.

33 Aukett MA, Parks YA, Scott PH, Wharton BA. Treatment with iron increases weight gain and psychomotor development. Archives of Disease in Childhood 1986; 61: $849-57$.

34 Soewondo S, Husaini M, Pollitt E. Effects of iron deficiency on attention and learning processes in preschool children: Bandung, Indonesia. American Journal of Clinical Nutrition 1989; 50: 667-74.

35 Walter T, De Andraca I, Chadud P, Perales CG. Iron deficiency anemia: adverse effects on infant psychomotor development. Pediatrics 1989; 84: 7-17.

36 Soemantri AG. Preliminary findings on iron supplementation and learning achievement of rural Indonesian children. American Journal of Clinical Nutrition 1989; 50 : 698-702.

37 Idjradinata P, Pollitt E. Reversal of developmental delays in iron deficient anaemic infants treated with iron. Lancet 1993; 341: $1-4$. 
38 Moffatt MEK, Longstaffe S, Besant J, Dureski C. Prevention of iron deficiency and psychomotor decline in high risk infants through use of iron fortified infant formula a randomised clinical trial. Journal of Pediatrics 1994; 125 : $527-34$.

39 Morley R, Abbott R, Fairweather-Tait S, Macfayden U, Stephenson T, Lucas A. Iron fortified follow on formula from 9 to 18 months improves iron status but not development or growth: a randomised trial. Archives of Disease in Childhood 1999; 81: 247-52.

40 Stoltzfus RJ, Kvalsvig JD, Chwaya HM, Montresor A, Albonico M, Tielsch JM, et al. Effects of iron supplementation and anthelminthic treatment on motor and language development of preschool children in Zanzibar: double blind, placebo controlled study. British Medical Journal 2001; 323: 1-8.

41 Stoltzfus RJ, Dreyfuss ML. Guidelines for the Use of Iron Supplements to Prevent and Treat Iron Deficiency Anemia. Washington, DC: ILSI Press, 1998.

42 Nestel P, Davidsson L. Anemia, Iron Deficiency, and Iron Deficiency Anemia. Washington, DC: ILSI Press, 2002.
43 Nokes C, van den Bosch C, Bundy DAP. The Effects of Iron Deficiency and Anaemia on Mental and Motor Performance, Educational Achievement, and Behaviour in Children: An Annotated Bibliography, Report of the International Nutritional Anemia Consultative Group (INACG). Washington, DC: INACG, 1998

44 Symes AL, Missala K, Sourkes TL. Iron and riboflavin dependent metabolism of a monoamine in the rat in vivo. Science 1971; 174: 153-5.

45 Dallman PR, Siimes M, Manies EC. Brain iron: persistent deficiency following short-term iron deprivation in the young rat. British Journal of Haematology 1975; 31: 209-15.

46 Findlay E, Reid RL, Ng KT, Armstrong SM. The effect of iron deficiency during development on passive avoidance learning in the adult rat. Physiology \& Behavior 1981; 27: 1089-96.

47 Weinberg J, Levin S, Dallman PR. Long term consequences of early iron deficiency in rat. Pharmacology, Biochemistry, and Behavior 1979; 11: 631-8. 